\title{
Molecular Characterization of two Maize Hybrids Based on Primer Bias
}

\author{
${ }^{1}$ Ehab R. El-Helow, ${ }^{1}$ Reem Badr, ${ }^{2,3}$ Gamal Osman and ${ }^{1}$ Amani Abdel-latif
}

${ }^{1}$ Department of Botany and Microbiology, Faculty of Science, Alexandria University. Egypt.

${ }^{2}$ Department of Biology, Faculty of Applied Science, Umm Al-Qura University, Makka, Saudi Arabia.

${ }^{3}$ Microbial Genetics Department, Agricultural Genetic Engineering Research Institute (AGERI), Giza, Egypt.

Correspondence Author: Prof. Dr. Gamal Osman. Department of Biology, Faculty of Applied Science, Umm Al Qura University, Makkah 21955, Saudi Arabia.

Tel: +0530760365

Received date: 22 December 2017, Accepted date: 22 January 2018, Online date: 5 February 2018

Copyright: (C) 2018 Ehab R. El-Helow et al. This is an open-access article distributed under the terms of the Creative Commons Attribution

\begin{abstract}
The DNA sequences in the internal transcribed spacer (ITS) of the nuclear ribosomal DNA showed great discriminatory power in angiosperms. However, a common complication in phylogenetic studies is the existence of two allele sequences with variable nucleotide sites for each locus in a diploid. The direct sequencing of polymerase chain reaction (PCR) products frequently results in a composite chromatogram for each individual organism. Therefore, various methods have been investigated to obtain single haplotype sequences. For molecular characterization of the two maize (Zea mays) hybrids, M10 and M321, high-quality genomic DNA was extracted from the grains using a modified Mericon extraction method. The universal primers, ITS1 and ITS4, were used in PCR for the amplification of alternative ITS alleles in each DNA sample. Among 2 ITS primers that were examined, DNA sequencing using ITS1and ITS1.1 primers resulted in a complex chromatogram with two or more homologous ITS sequences of each genome. However, ITS4revealed a single haplotype sequence for each strain. On the basis of Basic Local Alignment Search Tool similarity search, each of the two obtained allele sequences revealed a pattern characteristic to its strain. Along with closely related ITS haplotypes of other strains of Zea mays that are available at National Centre for Biotechnology Information databases, the two obtained sequences were aligned and phylogenetically analyzed. On the basis of primer bias, we suggest that studying known alternative ITS primers for the direct sequencing of pooled PCR products facilitate in identifying at least one primer that can generate a single ITS haplotype sequence from a diploid genome. Primer biases are investigated on the basis of the quality of seanence outnut
\end{abstract}

Key words :Zea mays, ITS, internal transcribed spacer, primer bias and haplotype

\section{INTRODUCTION}

The taxonomic difficulty for several systematics, field ecologists, and evolutionary biologists is correctly determining an organism by a rapid, repeatable, and reliable method [1]. Advances in molecular genetics, sequencing technology, and bioinformatics have explored numerous molecular markers that can be easily sequenced and used for inferring phylogenetic relationships. One of these markers is the internal transcribed spacer (ITS), which is part of the non-coding nuclear ribosomal DNA cistron. It consists of the regions ITS1 and ITS2 that are separated by the gene 5.8S. The ITS region displays high rates of substitution that can manifest species and even subspecies divergences $[2,3]$. Therefore, the ITS sequence is one of the most frequently used DNA markers in plant phylogenetic and DNA barcoding analyses [4, 5]. In addition, some genetic incidents exist that may mislead ITS-based phylogenetic investigations [6-8]. These incidents include the following: (a) existence of two alleles that may be heterozygous for each nuclear gene, (b) presence of several 18S-5.8S-26S repeats at one or more chromosomal locations, (c) some of these copies degenerate into pseudogenes, and (d) the near universality of most primers commonly used for the polymerase chain reaction (PCR) amplification of ITS sequences. Therefore, the direct sequencing of PCR-amplified ITS sequences would typically produce a mixture of allelic sequences with polymorphic nucleotide sites, which will appear as overlapping peaks. A nucleotide sequence obtained from such a mixed PCR product is consensus for several targets sharing the same priming sites in a single genome [9]. By contrast, DNA sequence data from an individual allele (called a haplotype) can provide considerable information to address various biological questions and to guide several practical applications [7, 10]. Various methods have been investigated to obtain haplotype sequences from different genomes. A traditional method is to construct a random cloning library from the PCR products before sequencing of the target fragment [7]. Furthermore, it has been suggested that reducing the amount of genomic DNA and decreasing the number of PCR cycles [11]. In addition to using different PCR primer sets and reaction conditions result in allele bias $[3,12,13]$. Maize (Zea mays) is one of the most productive and widely grown crops worldwide. It has remarkable morphological and genetic diversity and is considered a foundational model for studying genetics and genomics. An improved de novo assembly and annotation of a maize reference genome have been recently conducted [14]. To investigate the wide diversity of maize genotypes, few studies on ITS sequencing $[15,16]$ and microsatellites or simple sequence repeats $[17,18]$ have been published. The phylogenetic relationships of Zea species inferred from ITS sequences were demonstrated to be highly concordant with Random Amplified Polymorphic DNA (RAPD) evidence [16, 19]. The main objective of this study was to identify a molecular marker or a haplotype-specific signature sequence that can be used to distinguish the genomes of two Egyptian maize hybrids, M10 and M321M321. However, it is typically quite difficult to extract and purify DNA from cereals because of the presence of acidic polysaccharides, proteins, and DNA polymerase inhibitors such as tannins, alkaloids, and polyphenols in the extracts [20, 21]. The presence of these compounds reduces the quality and quantity of DNA, thus often making the sample non-amplifiable. Therefore, DNA of each maize hybrid was extracted from grains using a modified Mericon extraction method that has recently been demonstrated as a highly efficient technique [22]. The ITS target sequences in the two genomes were amplified using 
PCR with universal ITS primers. Subsequently, the selective sequencing of a single ITS haplotype from each pooled PCR product was performed on the basis of primer bias.

\section{MATERIALS AND METHODS}

\subsection{Plant materials and DNA extraction:}

The grains of two hybrid Zea mays strains coded as M10 and M321 were obtained from the Crop Institute, Agricultural Research Center, Giza, Egypt and were used as the source of genomic DNA. The fungal contaminations of the grains were removed using sodium hypochlorite [23]. High yield of improved quality DNA was then isolated from the grains using the modified Mericon extraction method [22]. DNA concentration, purity (A260/A280 ratio), and absorbance ratio at $260 \mathrm{~nm}$ to $280 \mathrm{~nm}$ (A260/A230 ratio) were measured for each sample $(1 \mu \mathrm{L})$ using a Thermo Scientific NanoDrop 1000 Spectrophotometer (Thermo Scientific, Germany). DNA extracts were analyzed by agarose gel electrophoresis using 0.75\% agarose gel (SeaKem LE agarose, Cambrex, and gels for genomic and amplified DNA). Electrophoresis was performed using $1 \times$ Tris-Borate EDTA buffer containing $1 \mu \mathrm{g} / \mathrm{mL}$ of ethidium bromide and a constant voltage of $100 \mathrm{~V}$. GelPilot 100-base pair (bp) ladder (Qiagen) was used as DNA marker. DNA bands were visualized and images were obtained using Gel Doc XR+ Imaging system (Bio-Rad Laboratories Inc., Germany).

\subsection{Polymerase chain reaction amplification and nucleotide sequencing:}

For PCR, each DNA sample was diluted to a working concentration of $20 \mathrm{ng} / \mu \mathrm{L}$ and used as a template. The universal ITS primers used in this study have been developed by White et al., [24] and were synthesized and purified by MWG Biotech. These primers included ITS1 (5'-TCCGTAGGTGAACCTGCGG-3'), ITS1.1 (5'-GAACCTGCGGAAGGATCAT-3'), and ITS4 (5'-TCCTCCGCTTATTGATATGC-3'). The ITS regions (including ITS1, 5.8S gene, and ITS2) of each sample were amplified using PCR with a Peltier Thermal Cycler (Bio-Rad Laboratories Inc., Germany) using primers ITS1 and ITS4. PCRs were performed and thermal cycling conditions were analyzed [22]. The amplified products were purified using Qiagen PCR Purification kit following the manufacturer's protocol. However, at the end of the purification protocol, all purification columns were eluted for two sequential times instead of one to obtain DNA at high and low concentrations from each PCR product. Subsequently, the two concentrations of the amplified ITS regions were separately sequenced using the forward primers ITS1 and ITS1.1 in addition to reverse primer ITS4. Cycle sequencing was performed using $5.5 \mu \mathrm{L}$ of the purified PCR product and $1 \mu \mathrm{L}$ of the Terminator Ready Reaction Mix according to the protocol of Applied Biosystems [25]. The products were purified using a Sequencing Reaction Cleanup kit (Millipore, Billerica, USA) and sequenced in one direction on an ABI $3730 \times 1$ DNA analyzer (Biometra, Germany). The resulting sequences were edited and analyzed using BioEdit. Any nucleotide sequence that showed polymorphism was omitted. However, ITS sequences that lack polymorphic sites were only ex tracted from high-quality chromatograms.

\subsection{Sequence alignment and phylogenetic analysis:}

The ITS sequences obtained from M10 and M231 genomes were compared with the nucleotide collection of the National Centre for Biotechnology Information (NCBI) using the Basic Local Alignment Search Tool (BLAST). The boundaries of the entire ITS region were determined on the basis of homologous ITS sequences of Zea mays published in the GenBank database [2]. Subsequently, the experimental sequences were analyzed together with nine closely related ITS sequences of Zea mays. The proportion of nucleotide differences was calculated among pairs using ClustalW. In addition, the sequences were aligned using webPRANK, a phylogeny-aware progressive alignment program. The software MEGA 7.0 was then used for phylogenetic analysis of the obtained multiple sequence alignment (the entire ITS1-5.8S-ITS2 region), and to generate a phylogenetic tree using the maximum likelihood method. The tree was rooted using KU182535, a comparatively divergent Zea mays ITS sequence obtained from NCBI, as outgroup. The statistical support for internal nodes was estimated using 100 bootstrap samples [26].

Results:

\subsection{Assessment of the extracted DNA:}

As previously described, the quality of extracted DNA samples from each maize strain was verified spectrophotometrically using a NanoDrop instrument and agarose gel electrophoresis. The recorded $260 / 280 \mathrm{~nm}$ ratio (1.8) indicated that the extracted DNA was of high purity with the absence of proteins and phenols. Gel electrophoresis revealed a single band of high molecular weight DNA with slight evidence of shearing and absence of RNA or polysaccharide contamination.

\subsection{PCR amplification and sequencing of the internal transcribed spacer region:}

The universal primers ITS1 and ITS4 were used to amplify alternative alleles of the ITS region in the total genomic DNA of the two Zea mays strains, M10 and M321. Each of the two reactions resulted in a DNA amplicon of approximately $700 \mathrm{bp}$ when examined on an agarose gel. As described in the Materials and Methods section, each PCR product was purified and prepared at two different working concentrations before sequencing.

For each strain, the direct sequencing of the concentrated and diluted pooled PCR products using the forward primer ITS1 resulted in DNA fragments ranging between $644 \mathrm{bp}$ and $655 \mathrm{bp}$. All chromatograms showed a mixture of homozygous and heterozygous nucleotide sites along the entire sequence. Figure 1a shows a representative part of the obtained sequence outputs. These composite sequence profiles confirmed the presence of two or more ITS sequence patterns in each of the two experimental maize hybrids. In addition, similar results were achieved by using ITS1.1 (Figure 1b), which is an alternative forward primer that acts at $9 \mathrm{bp}$ downstream of ITS1. Moreover, it is crucial to emphasize on the corresponding sequences obtained from the two examined template DNA concentrations that resulted in chromatograms with slightly different consensus sequences and did not show clear variations with respect to their quality.

However, the reverse primer ITS4 showed the best sequencing results in all cases under the used experimental conditions (Figure 1c). In each of the obtained chromatograms of ITS sequences, the observed overlapping peaks were restricted to few sites that are located at short boundaries of the sequences. Therefore, pure allele sequences that lack polymorphic sites were simply extracted from the ITS4-based sequence outputs by editing. Interestingly, the two sequences obtained from both concentrated and diluted PCR amplicons of each strain using the reverse primer ITS4 were identical and confirmed each other. The edited ITS sequences resulting from M10 and M321 genomes were 553 bp and 551 bp in length and denoted as haplotypes Hm10.1 and Hm231.1, respectively. On the basis of BLAST search using NCBI databases, the edited ITS fragments showed that the length was matching with the boundaries of previously published homologous Zea mays ITS sequences [15]. The results of pairwise sequence alignment by BLAST demonstrated that none of the ITS nucleotide sequences of Zea mays available through NCBI databases showed $100 \%$ identity to any of the two obtained ITS haplotypes. Closely related ITS sequences, however, demonstrated a range of variations with at least $1 \%$ sequence dissimilarity. The Hm10.1 and Hm231.1 sequences were then submitted to the GenBank database under the accession numbers MF780725 and MF780726, respectively.

\subsection{Phylogenetic analysis of the obtained internal transcribed spacer haplotypes:}

Pairwise alignment of $\mathrm{Hm} 10.1$ and $\mathrm{Hm} 231.1$ showed that they are closely related. They showed a sequence divergence of approximately $0.91 \%$, which is limited to a single gap of two nucleotides in the ITS sequence of the haplotype Hm231.1. In particular, this gap is present in the ITS1 region, which is located between 18S and 5.8S rRNA. Subsequently, multiple sequence alignments were performed using the two new sequences together with a group of related Zea mays ITS haplotypes obtained through the BLAST search. The aligned fragments showed sequence divergence ranging between $0.19 \%$ and $2.41 \%$ and demonstrated the presence of both substitutions and indels (insertions and deletions) in the ITS region. For further analysis of the aligned ITS sequences, a phylogenetic tree was constructed using the maximum likelihood method and the tree was rooted using KU182535.1 ITS sequence as outgroup. The aligned sequences revealed three clusters with $72 \%, 58 \%$, and $55 \%$ bootstrap support as shown in Figure 2 . The two experimental haplotypes formed a single cluster with three previously published Zea mays ITS alleles [2] including U46601.1, U46612.1, and U46610.1. 
Discussion:

As there are several similarities in superficial characters and chemical compositions between closely related genotypes, it is difficult to identify hybrids of Zea mays by traditional identification methods. However, DNA barcoding has been proposed to be one of the most promising tools for accurate and rapid identification of taxa [27]. In particular, DNA sequences in the ITS of the nuclear ribosomal DNA show great discriminatory power in angiosperms when compared with other molecular chronometers such as plastid genome markers [28, 29]. The analysis of ITS sequences was performed for finding out a unique nucleotide signature that can be used for the identification of a specific genotype. In this study, we investigated the ITS region to identify variations in DNA sequences between the two strains, M10 and M321, and the other published Zea mays ITS haplotypes. However, one of the most prevalent complications for phylogenetic inference is the existence of extensive allelic variations in several plant genomes [2]. In sexual diploids, such as most plants and animals, including humans, each nuclear gene has two alleles where one allele comes from each parent. Therefore, the two alleles have different gene sequences and evolutionary histories [7]. Furthermore, the apparent heterozygotes can be a consequence of copy number variants and repeats [30]. Parallel to these considerations, the sequencing results of this study revealed that each PCR reaction leads to a pool of ITS allele sequences. This ITS polymorphism was noted in each individual strain in the composite chromatograms developed by direct sequencing with universal primers ITS1 and ITS1.1. In addition, this observation corresponds to the hybrid nature of the two examined genotypes. Under such a condition, methods to obtain single haplotype sequences have to be employed. [11] recommend reducing the amount of genomic DNA and decreasing the number of PCR cycles in PCR reactions that contain heterozygous ITS target sequences. However, corresponding PCR amplicons obtained from two template DNA concentrations that we studied resulted in low-quality chromatograms with slightly different consensus sequences. Another method is to construct a random cloning library from the PCR products before sequencing of the target locus in randomly chosen PCR clones [9]. However, this method is slow, tedious, and expensive because it involves routine sequencing of large numbers of individual PCR clones from each genomic DNA [2,3]. Alternatively, the most commonly used ITS primers are characterized by various types of biases such as length bias and primer mismatch bias [12]. Furthermore, this target bias is affected by the reaction conditions [2]. Therefore, it can be speculated that using alternative primer sequences and PCR and/or sequencing conditions may reduce the targets of the primer, and consequently, improve the obtained chromatograms. The results of this study demonstrated that direct sequencing of pooled PCR products using ITS4 resulted in much cleaner chromatograms when compared with those using ITS1 and ITS1.1. This observation suggests that the applied sequencing conditions considerably restrict the targets of the primer ITS4 and consequently, reveal a single ITS sequence from each genome. With this reverse primer, the change in template DNA concentration neither affected PCR amplification nor sequencing results. Therefore, the two sequencing results from each corresponding pair of PCR reactions confirmed each other and served as a sequence replica. The phylogenetic tree inferred from the new ITS sequences and their published close relatives indicated that the obtained data largely match with the published ITS sequences of Zea mays genotypes. The multiple sequence alignment of these homologous sequences demonstrated the presence of both insertions/deletions and substitutions including transitions and transversions in the ITS region. On the basis of BLAST similarity search using NCBI database, each of the obtained allele sequences revealed a pattern characteristic to its strain and can be considered haplotype-specific signature sequences for the genotypes of the maize strains, M10 and M321.

80

C C C G C G G G G AAG G G G G G G C G C G AAAAAAAAAC CA C G G C

(a)

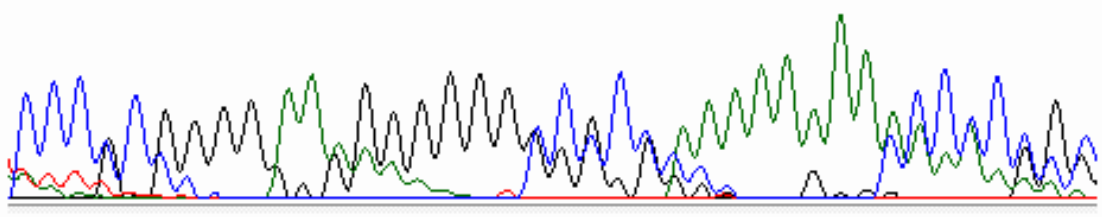

80

90

100

110

C C C G C G G G AAG G G G G G G C G C G AAAAAAAAAC C C C C G G

(b)

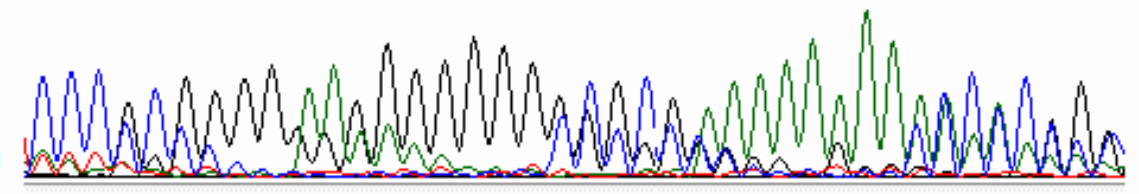

510

520

530

540

C C G T G G T T C T T T T T C G G G C C C C C C T T C C C C C G G G

(c)

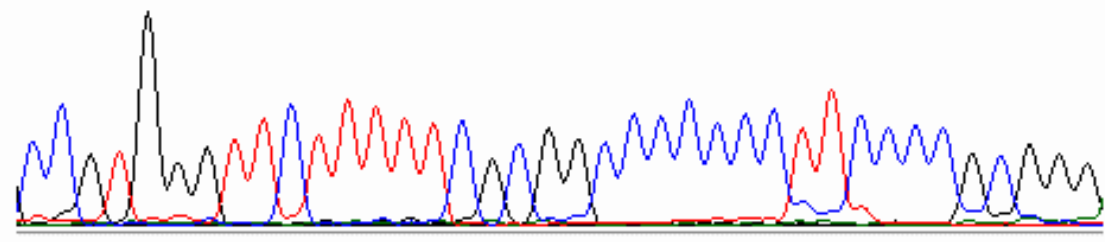

Fig. 1: Sequencing chromatograms of representative corresponding parts from the ITS sequences obtained using pooled PCR products of M321 in the presence of the forward primers ITS-1 (a) and ITS-1.1 (b) and the reverse primer pITS-4 (c). 

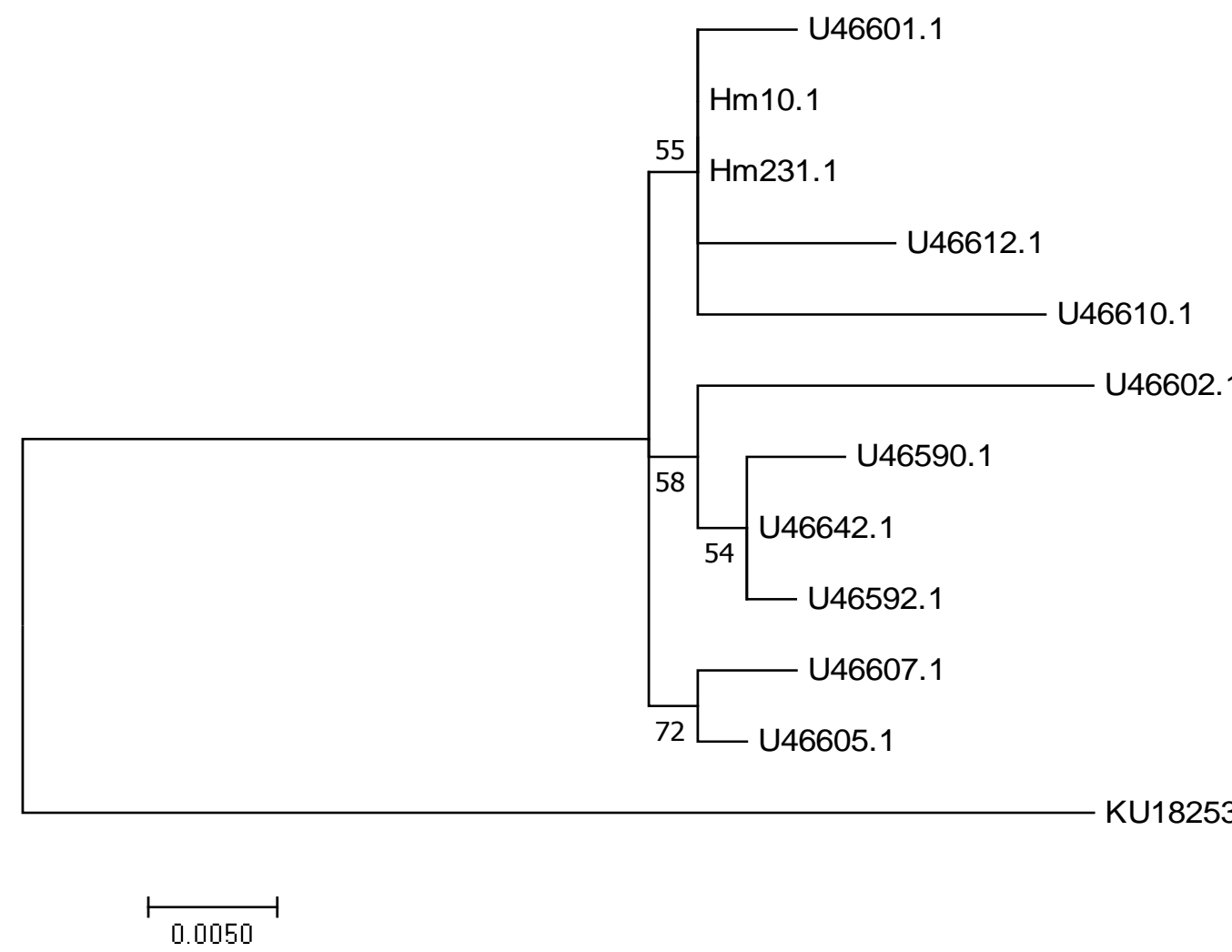

Fig. 2: The maximum likelihood phylogenetic tree generated on the basis ofZea mays ITS haplotypes including the obtained Hm10.1 and Hm231.1 sequences together with nine closely related sequences in addition to KU182535 as a outgroup. The branch-lengths are proportional to the amount of sequence divergence and the number of above branches indicating bootstrap values from 100 replications.

\section{Conclusion:}

We suggest that alternative universal primers designed for a specific target in a diploid genome can separately be studied for the direct sequencing of pooled PCR products to identify primers with restricted target recognition under a sequencing condition. Such primer biases are assessed on the basis of the output of sequencing chromatograms. The results of this study demonstrated that the reverse primer ITS4 can be usedto obtain a single ITS haplotype sequence from each of two examined Zea maysgenomes.On the basis of our results that have been validated by BLAST analysis, the new ITS sequences can facilitate in distinguishing M10 and M321genotypes from other maize strains.

\section{ACKNOWLEDGEMENTS}

The Authors acknowledge Faculty of Science, Department of Botany and Microbiology, Alexandria University, Egyptand Department of Biology, Faculty of Applied Science, Umm Al-Qura University, Kingdom of Saudi Arabia for supporting this research.

\section{REFERENCES}

[1] Janzen, D., 2005. In: "How to conserve wild plants? Give the world the power to read them. In: Krupnick, G.A., W.J .Kress, (eds) "Plant conservation: a natural history approach". University of Chicago Press, Chicago.

[2] Buckler, E., T. Holtsfoot, 1996. "Zea systematics: ribosomal ITS evidence" Mol. Biol. Evol , 13:612-622.

[3] Baldwin, B., M. Sanderson., J. Porter., M. Wojciechowski., C. Campbell., M. Donoghue, 1995. "The ITS region of nuclear ribosomal DNA-A valuable source of evidence on Angiosperm phylogeny" Ann. Mo. Bot. Gard, 82: 247-277.

[4] Cheng, H., 2016. "The F-box Protein Rcy1 Is Involved in the Degradation of Histone H3 Variant Cse4 and Genome Maintenance" J Biol Chem, 291(19):10372-7.

[5] Eissa, H. F., S. E. Hassanien., A.M. Ramadan., M. M. El-Shamy., O. M. Saleh., A. M. Shokry., M. Abdelsattar., Y. B. Morsy., M. A. El-Maghraby., H. F. Alameldin., S. M. Hassan., G. H. Osman., H. T. Mahfouz., G. A. Gad El-Karim., M. A. Madkour., A. Bahieldin, 2017. "Developing transgenic wheat to encounter rusts and powdery mildew by overexpressing barley chi26 gene for fungal resistance" Plant Methods, 13:41.

[6] Alvarez. I., J. Wendel, 2003. "Ribosomal ITS sequences and plant phylogenetic inference" Mol. Phylogenet. Evol, 29:417-434

[7] Xu, J., 2006. "Extracting haplotypes from diploid organisms" Current issues in molecular biology, 8(2): 113.

[8] Abdel Khalik, K., G. Osman, 2017. "Genetic analysis of Plctranthus L. (Lamiaceae) in Saudi Arabia based RAPD and ISSR markers" Pakistan Journal of Botany, 49:1073-1084.

[9] Feliner, G., J. Rossello, 2007. "Better the devil you know? Guidelines for insightful utilization of nrDNA ITS in species-level evolutionary studies " Plants Molecular Phylogenetics and Evolution, 44: 911-919.

[10] Abouseadaa, H., G. Osman., A, Ramadan., S. Hassanein., M. Abdelsattar., Y. Morsy., H. Alameldin., D. El-Ghareeb., H. Nour-Eldin., R. Salem., A. Gad., S. Elkhodary., M. Shehata., H. Mahfouz., H. Eissa., A. Bahieldin, 2015. "Development of transgenic wheat (Triticum aestivum L.) expressing avidin gene conferring resistance to stored product insects" BMC Plant Biology, 15:183-190.

[11] Wagner, A., P. Blackstone., M. Cartwright, B. Dick., P. Misof., G. Snow., J. Wagner., M. Bartels., J. Pendleton, 1994. "Surveys of gene families using polymerase chain reaction: PCR selection and PCR drift" Syst. Biol , 43:250-261.

[12] Bellemain, E., T. Carlsen. , C. Brochmann., E. Coissac., P. Taberlet., H. Kauserud, 2010. "ITS as an environmental DNA barcode for fungi: an in silico approach reveals potential PCR biases" BMC Microbiology, 10(1):189. 
[13] Osman, G., S. K. Assem., R. M. Alreedy ., D. K. El-Ghareeb., M. A. Basry., A. Rastogi., H. M. Kalaji, 2015. "Development of insect resistance maize plants expressing the chitinase gene of Spodoptera littoralis" Nature: Scientific Reports, 5:18067 |DOI: 10.1038/srep18067.

[14] Jiao, W., G . Accinelli., B. Hartwing., C. Kiefer., D. Baker, 2017. "Improving and correcting the contiguity of long-read genome assemblies of three plant species using optical mapping and chromosome information capture data" Genome Res, Doi:10.1 101/gr.213625.116.

[15] Buckler, E., T. Holtsfoot, 1996. "Zea ribosomal repeat evolution and substitution patterns" Mol. Biol. Evol, 13:623-632.

[16] Wang, P., Y. Lu., M. Zheng., T. Rong., Q. Tang, 2011. "RAPD and internal transcribed spacer sequence analyses reveal Zea nicaraguensis as a section Luxuriantes species close to Zea luxurians" PLoS One, 6; 4: e16728.

[17] Smith, J., L. Chin., H. Shu., O. Smith., S. Wall., M. Senior., S. Mitchell., S. Kresovich., J. Zieglem, 1997. "An evaluation of the utility of SSR loci as molecular markers in maize (Zea mays L.): comparisons with data from RFLPs and pedigree." TAG Theoretical and Applied Genetics, 95(1):163-173.

[18] Matsuoka. Y, Y. Vigouroux., M. Goodman., J. Sanchez., E. Buckler., J. Doebley, 2002. "A single domestication for maize shown by multilocus microsatellite genotyping" Proceedings of the National Academy of Sciences, 99(9):6080-6084.

[19] Abdel Khalik, K., G. Osman., W. Al-Amoudi, 2012. "Genetic diversity and relationships of some Ipomoea species based on analysis of RAPD-PCR and SDS-PAGE of seed proteins" AJCS, 6 (6):1088-1093.

[20] Sarwat, M., M. Negi., M. Lakshmikumaran., A. Tyagi., S. Das., P. Srivastava, 2006. "A standardized protocol for genomic DNA isolation from Terminalia arjuna for genetic diversity analysis" Electron. J. Biotechnol, 9:86-91.

[21] Munshi, A., G. Osman, 2010. "Investigation on molecular phylogeny of some date palm (Phoenix dactylifra L.) cultivars by protein, RAPD and ISSR markers in Saudi Arabia" AJCS, 4 (1): 23-28.

[22] Abdel-latif, A., G. Osman, 2017. "Comparison of three genomic DNA extraction methods to obtain next-generation sequencing quality DNA from maize" Plant Methods, 13:1.

[23] López, M., J. Peralta-Videa., T. Benitez., J. Gardea-Torresdey, 2005. "Enhancement of lead uptake by alfalfa (Medicago sativa) using EDTA and a plant growth promoter" Chemosphere, 61(4): 595-598.

[24] White, T., T. Bruns., S. Lee., J. Taylor, 1990. "Amplification and direct sequencing of fungal ribosomal RNA genes for phylogenetics" In: M. Innis, D. Gelfand, J. Sninsky \& T. White (Eds.), PCR protocols. A guide to methods and applications. New York: Academic Press, 315-322.

[25] Applied Biosystems., 2002. BigDye® terminator v1.1 cycle sequencing kit protocol. Foster City: Applied Biosystems.

[26] Felsenstein., J, 1985. "Confidence limits on phylogenies" An approach using the bootstrap. Evolution, 39:783-791.

[27] Xu, S., D. Li., J. Li., X. Xiang., W. Jin., W. Huang., X. Jin., L. Huang, 2015. "Evaluation of the DNA barcodes in Dendrobium (Orchidaceae) from mainland Asia, PLoS One, 10: e0115168.

[28] Yao., Y, 2010. "Ecm1 is a new pre-ribosomal factor involved in pre-60S particle export. RNA" 16(5):1007-17.

[29] Li, D., L. Gao., H. Li., H. Wang., X. Ge., J . Liu, 2011. "Comparative analysis of a larger dataset indicates that internal transcribed spacer (ITS) should be incorporated into the core barcode for seed plants" PNAS 108(49): 19641-19646.

[30] Noyes, H., D. Daly, I. Goodhead, S. Kay., S. Kemp, J. Kenny, N. Hall, 2015. "A simple procedure for directly obtaining haplotype sequences of diploid genomes" BMC genomics, 16 (1):642. 